\title{
Alleviation of norflurazon-induced photobleaching by overexpression of Fe-chelatase in transgenic rice
}

\author{
Joon-Heum Park and Sunyo Jung* \\ School of Life Sciences, BK21 FOUR KNU Creative BioResearch Group, Kyungpook National University, Daegu 41566, Korea \\ (Received March 24, 2021; Accepted June 10, 2021)
}

We examined the effect of Bradyrhizobium japonicum FeCh (BjFeCh) expression on the regulation of porphyrin biosynthesis and resistance to norflurazon (NF)-induced photobleaching in transgenic rice. In response to NF, transgenic lines F4 and F7 showed lesser declines in chlorophyll, carotenoid, $F_{\mathrm{v}} / F_{\mathrm{m}}, \phi_{\mathrm{PS} I}$, and light-harvesting chlorophyll (Lhc) a/b-binding proteins as compared to wild-type (WT) plants, resulting in the alleviation of NF-induced photobleaching. During photobleaching, levels of heme, protoporphyrin IX (Proto IX), Mg-Proto IX (monomethylester), and protochlorophyllide decreased in WT and transgenic plants, with lesser decreases in transgenic plants. Most porphyrin biosynthetic genes were greatly downregulated in WT and transgenic plants following NF treatment, with higher transcript levels in transgenic plants. The expression of $\mathrm{BjFeCh}$ in transgenic rice may play a protective role in mitigating NF-induced photobleaching by maintaining levels of heme, chlorophyll intermediates, and Lhc proteins. This finding will contribute to understanding the resistance mechanism of NF-resistant crops and establishing a new strategy for weed control.

Keywords: chlorophyll fluorescence, Fe-chelatase, heme, photobleaching, porphyrin.

\section{Introduction}

In photosynthetic organisms, porphyrins play essential roles in the conversion of light to chemical energy (photosynthesis) and the electron transfer processes (metabolism). ${ }^{1-4)}$ In the last common step of the porphyrin biosynthetic pathway, the conversion of protoporphyrinogen IX to protoporphyrin IX (Proto IX) leads to the biosynthesis of heme and chlorophyll (Fig. 1). ${ }^{1)}$ The first step in the heme branch of the pathway is the insertion of $\mathrm{Fe}^{2+}$ into Proto IX, a reaction catalyzed by $\mathrm{Fe}$-chelatase ( $\mathrm{FeCh}, \mathrm{EC}$ 4.99.1.1), whereas in the chlorophyll branch, Mg-chelatase (EC 6.6.1.1) catalyzes the insertion of $\mathrm{Mg}^{2+}$ into Proto IX to produce chlorophyll. ${ }^{1,45}$ Excess Proto IX and protochlorophyllide (Pchlide) are strong photosensitizers that generate reactive oxygen species (ROS), such as $\mathrm{H}_{2} \mathrm{O}_{2}$ and ${ }^{1} \mathrm{O}_{2}{ }^{4,6)}$ The deregulation of

\footnotetext{
* To whom correspondence should be addressed.

E-mail: sjung@knu.ac.kr

Published online August 31, 2021
}

(c) BY-NC-ND () Pesticide Science Society of Japan 2021. This is an open access article distributed under the Creative Commons Attribution-NonCommercial-NoDerivatives 4.0 International (CC BY-NC-ND 4.0) License (https://creativecommons.org/licenses/by-nc-nd/4.0/) metabolic flux in the porphyrin biosynthetic pathway by stress conditions, such as drought or extreme temperatures, results in severe plant damage. ${ }^{7,8)}$

Chlorophyll, the most abundant porphyrin in plants, is bound to the light-harvesting chlorophyll (Lhc) $a / b$-binding proteins and is in direct contact with carotenoids that protect the photosynthetic apparatus by quenching harmful $\operatorname{ROS}\left({ }^{1} \mathrm{O}_{2}\right)$ produced by overexcitation of chlorophyll. ${ }^{9-11)}$ The bleaching herbicide norflurazon (NF) inhibits phytoene desaturase activity, leading to phytoene accumulation at the expense of colored cyclic carotenoids. ${ }^{12,13)} \mathrm{NF}$ prevents the formation of enough carotenoids to ensure efficient photoprotection of the photosynthetic apparatus. Consequently, chlorophyll is degraded, which causes the typical bleaching symptoms and inhibits plastid biogenesis in NF-treated plants. ${ }^{12,14)}$ Thus, this perturbation can generate excess ROS and necessitate additional defense mechanisms. Under photooxidative stress conditions, plants must dissipate excess light energy to protect the photosynthetic apparatus from photooxidative destruction. ${ }^{9,15)}$ Non-photochemical quenching (NPQ) of chlorophyll fluorescence is considered to be a reflection of processes in the photosynthetic membrane that are not photochemical in origin. ${ }^{16)}$

Previous studies have shown the induction of $\mathrm{FeCh}$ mRNA in response to NF treatment. ${ }^{17,18)}$ In the present study, we used an 


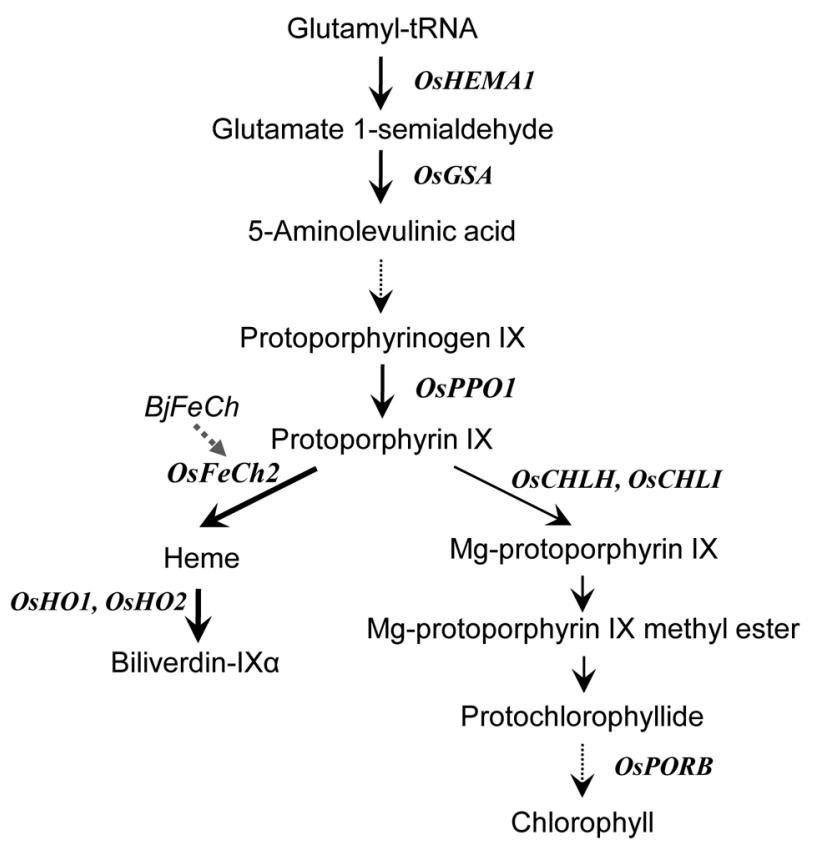

Fig. 1. The porphyrin biosynthetic pathway in plants showing the genes analyzed in this study. Gene names: OsHEMA1, glutamyl-tRNA reductase 1; OsGSA, glutamate 1-semialdehyde aminotransferase; OsPPO1, protoporphyrinogen oxidase 1; $\mathrm{OsFeCh} 2$, Fe-chelatase 2; OsHO1, heme oxygenase 1; $\mathrm{OsHO}$, heme oxygenase 2; $\mathrm{OsCHLH}, \mathrm{H}$-subunit of $\mathrm{Mg}$-chelatase; OsCHLI, I-subunit of Mg-chelatase; OsPORB, protochlorophyllide oxidoreductase $\mathrm{B} ; \mathrm{BjFeCh}$, Bradyrhizobium japonicum $\mathrm{Fe}$-chelatase. The overexpression of $\mathrm{BjFeCh}$ in transgenic rice is shown with a dashed arrow. Dotted arrows indicate multiple enzymatic steps. Increased flux in the heme branch is shown with thick solid arrows.

approach for $\mathrm{FeCh}$ overexpression to investigate the role of $\mathrm{FeCh}$ in NF-induced photobleaching. The Bradyrhizobium japonicum $\mathrm{FeCh}(\mathrm{BjFeCh})$ gene was chosen to prevent the co-suppression of homologous $\mathrm{FeCh}$ genes in transgenic rice (Oryza sativa). We examined whether the heterologous expression of $\mathrm{BjFeCh}$ in rice can increase resistance to NF-induced photobleaching. To address this question, we compared differential photodamage with respect to the photosynthetic efficiency and integrity of photosynthetic complexes in wild-type (WT) and transgenic plants treated with NF. We also analyzed the metabolic profiles and expression of key genes in porphyrin biosynthesis during NF-induced photobleaching.

\section{Materials and methods}

\section{Plant material and norflurazon (NF) treatment}

To test resistance to norflurazon (NF)-induced photobleaching, seeds from WT rice (Oryza sativa cv. Nakdong) and Bradyrhizobium japonicum $\mathrm{Fe}$-chelatase ( $\mathrm{BjFeCh}$ )-expressing transgenic rice lines (F4 and F7) ${ }^{19)}$ were placed on half-strength Murashige and Skoog (MS) medium containing various concentrations (0.1-2 $\mu \mathrm{M}$ ) of technical-grade NF (Sigma-Aldrich, St. Louis, MO, USA), and maintained in darkness for 2 day. Then, the germinated rice seedlings were grown for 5 day in a growth room maintained at day/night temperatures of 28 and $25^{\circ} \mathrm{C}$, respec- tively, under a $14 \mathrm{hr}$ light $/ 10 \mathrm{hr}$ dark photoperiod, with a photosynthetic photon flux density of $200 \mu \mathrm{mol} \mathrm{m}^{-2} \mathrm{~s}^{-1}$. At concentrations above $1.5 \mu \mathrm{M}$ NF, both WT and transgenic plants showed severe photobleaching symptoms. Therefore, we selected a concentration of $1 \mu \mathrm{M} \mathrm{NF}$ for further physiological experiments. After 5 day of exposure to the photoperiods, leaves from untreated control and NF-treated WT and transgenic plants were sampled $4 \mathrm{hr}$ after the start of the light phase.

\section{Measurement of photosynthetic activity}

Chlorophyll $a$ fluorescence was measured in vivo using a pulse amplitude modulation fluorometer (JUNIOR-PAM; Walz, Effeltrich, Germany) after dark adaptation for $20 \mathrm{~min}$. The ratio of $F_{\mathrm{v}}$ to $F_{\mathrm{m}}$, representing the activity of photosystem (PS) II, was used to assess the functional damage to plants. ${ }^{20)}$ The quantum yield of electron transport through PSII $\left(\phi_{\mathrm{PSII}}=\Delta F / F_{\mathrm{m}}^{\prime}: \Delta F=F_{\mathrm{m}}^{\prime}-F_{\mathrm{t}}\right.$; $F_{\mathrm{m}}^{\prime}$, maximal chlorophyll fluorescence in light-adapted state; $F_{\mathrm{t}}$, chlorophyll fluorescence in steady state), which measures the quantum efficiency of PSII, was calculated as defined by Genty et al. ${ }^{20)}$ The quantum yield of PSII provides a quick and useful estimate of the light-reaction activity and photosynthetic rate. NPQ was quantified according to the Stern-Volmer equation, NPQ= $F_{\mathrm{m}} / F_{\mathrm{m}}^{\prime}-1$, as previously described by Bilger and Björkman. ${ }^{21)}$

\section{Protein extraction, SDS-PAGE, and immunoblot analysis}

Leaf tissues were ground in liquid nitrogen and extracted with a buffer containing $2 \%(\mathrm{w} / \mathrm{v})$ SDS, $56 \mathrm{mM} \mathrm{Na}_{2} \mathrm{CO}_{3}, 12 \%(\mathrm{w} / \mathrm{v})$ sucrose, $56 \mathrm{mM}$ dithiothreitol (DTT), and $2 \mathrm{mM}$ ethylenediaminetetraacetic acid (EDTA), pH 8.0, followed by sonication. The mixture was centrifuged at $12,000 \mathrm{~g}$ for $20 \mathrm{~min}$ at $4^{\circ} \mathrm{C}$, and the resulting supernatants were collected to obtain total soluble proteins. BjFeCh proteins were separated on a $12 \%$ SDS-PAGE gel and Lhc proteins on a 14\% SDS-PAGE gel, and the proteins were electroblotted onto PVDF membranes. The expressions of antenna proteins of PSI (Lhca2 and Lhca3) and the major lightharvesting proteins of PSII (Lhcb1 and Lhcb2) were assayed with anti-Lhca and anti-Lhcb antibodies (Agrisera, Vännäs, Sweden). A polyclonal mouse antiserum for $\mathrm{BjFeCH}$ was raised against the purified recombinant $\mathrm{FeCh}$ protein of $B$. japonicum (Anygene Co., Gwangju, Korea). Immunodetection was performed according to standard procedures (F. Hoffmann-La Roche, Basel, Switzerland).

\section{RNA extraction and $q R T-P C R$}

Total RNA was prepared from leaf tissues using TRIzol Reagent (Invitrogen, Carlsbad, CA, USA) in accordance with the manufacturer's instructions, and $5 \mu \mathrm{g}$ of RNA from each sample was used for the reverse transcription reaction ( $\mathrm{Su}-$ perScript III First-Strand Synthesis System, Invitrogen). The resultant cDNAs were used as templates for qRT-PCR, using Actin as an internal control. qRT-PCR analysis was carried out using the StepOnePlus Real-Time PCR system (Applied Biosystems, Waltham, MA, USA) with Power SYBR Green PCR Master Mix (Applied Biosystems) and gene-specific prim- 
ers. The following gene-specific primers were used for PCR amplification: 5'-GTG GAC GCA TCA AAA TTG C-3' and 5'-GGG TTC GCT GTC GTT GAG-3' for $\mathrm{BjFeCh}$, 5'-GCT ATG GGT GGT GTT CGA CT-3' and 5' -CGA TCT TCT GGA GGC ACT TC-3' for OsHEMA1, 5' -CTC CGT GAC TTG ACG AAA CA- $3^{\prime}$ and $5^{\prime}$-GTA GGT TCC AGG CTCCATCA-3' for OsGSA, $5^{\prime}$-ACA GTT CCT CAT TGG CCA TC- ${ }^{\prime}$ ' and 5' -CCC ATG AAA TTT TTG CTG CT-3' for OsPPO1， 5' -GTG TGG GTT GCG TTC TTT TT- ${ }^{\prime}$ and $5^{\prime}$-GGT GAC AAT GTG GCT CCT CT-3' for OsCHLH, 5' $5^{\prime}$-TGT GCT TCT GGA TTC TGC TG-3' and 5'-GCT GGA GCT TGT CTT GTT CC-3' for OsCHLI, 5' -GTG AAT TGC CAG GTT TTC GT- $3^{\prime}$ and $5^{\prime}$-GCA ATT AGC AAA GCT GCA CA-3' for OsPORB, 5' -TTGGTG CTA TGG CAG TTT CA-3' and 5' ${ }^{\prime}$-AGT GGA ACA AAG GCA GGA TG-3' for OsFeCh2, 5' -AGC GCT AGC AGT AGC AGG AG-3' and $5^{\prime}$ GCT CCT TCT CCC CTT CCT T-3' for OsHO1, 5' -AGG GAC CTA GCA GCC CTA AC-3' and 5' -CCC GTA TCG TCC ATC TTG AG-3' for OsHO2, 5' -CAG CTC TCA CAG CTC ACT GC$3^{\prime}$ and 5'-GTG TCC CACCCG TAG TCG-3' for OsLhcb1, 5' ACA ACG AAG CCA GCT AAA ACG-3' and 5' -CCC GCA CCG ATT TAT TGG-3' for OsLhcb2, and 5' -CTT CAT AGG AAT GGA AGC TGC GGG TA-3' and 5' -CGA CCA CCT TGA TCT TCA TGC TGC TA- $3^{\prime}$ for OsActin.

\section{Determination of porphyrin contents}

Plant tissue was ground in methanol:acetone: $0.1 \mathrm{~N} \mathrm{NaOH}$ $(9: 10: 1,[\mathrm{v} / \mathrm{v}])$, and the homogenate was centrifuged at $10,000 \mathrm{~g}$ for $10 \mathrm{~min}$ at $4^{\circ} \mathrm{C} .^{22)}$ Porphyrin was separated by HPLC using a Nova-Pak $\mathrm{C}_{18}$ column (Waters, Milford, MA, USA) and eluted with a solvent system of $0.1 \mathrm{M}$ ammonium phosphate $(\mathrm{pH}$ 5.8) and methanol. The column eluate was monitored using a fluorescence detector (2474, Waters) at excitation and emission wavelengths of 400 and $630 \mathrm{~nm}$ for Proto IX, 440 and $630 \mathrm{~nm}$ for Pchlide, and 415 and $595 \mathrm{~nm}$ for Mg-Proto IX and Mg-Proto IX monomethylester (ME), respectively. The chlorophyll and carotenoid contents were spectrophotometrically determined according to the method of Lichtenthaler. ${ }^{23)}$

Heme was extracted as described previously. ${ }^{24)}$ Acid-acetone extracts were extracted again with diethyl ether and put on a QMA SepPak column (Waters). Protoheme was further purified and separated by HPLC on a Nova-Pak $\mathrm{C}_{18}$ column (Waters) with a solvent system of ethanol: acetic acid: $\mathrm{H}_{2} \mathrm{O}(66.5: 17: 16.5$, [v/v]) and detection at $402 \mathrm{~nm}$.

\section{Results}

1. Resistance of transgenic rice expressing BjFeCh to norflurazoninduced photobleaching

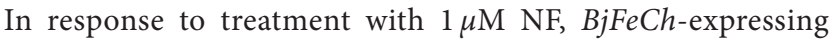
transgenic lines, F4 and F7, exhibited pale green shoots, whereas WT plants exhibited white shoots (Fig. 2). To estimate the inhibition of carotenoid biosynthesis by NF, we measured the total carotenoid content in NF-treated WT and transgenic plants. Carotenoid contents decreased by $87 \%$ in response to NF treatment in WT plants, but only by $70 \%$ and $61 \%$ in transgenic lines $\mathrm{F} 4$
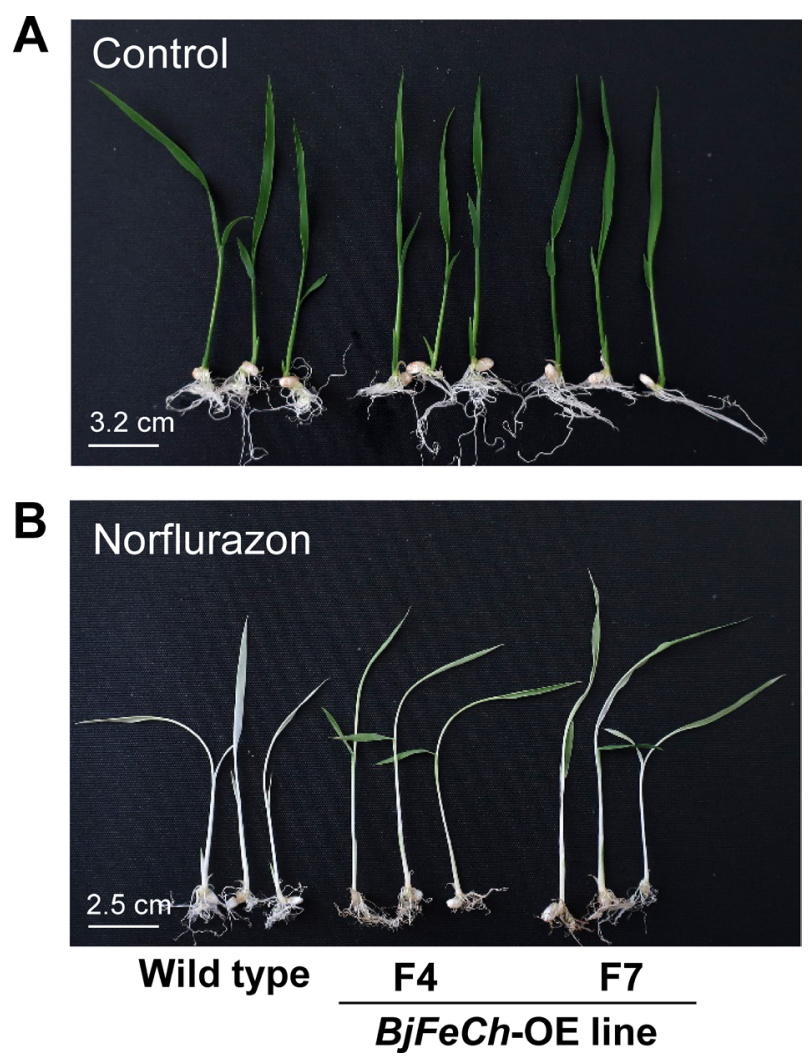

Fig. 2. Norflurazon-induced photobleaching in wild-type (WT) and transgenic rice expressing $\mathrm{BjFeCh}$ without (A) and with norflurazon treatment (B). WT and transgenic rice seedlings were grown without and with $1 \mu \mathrm{M}$ NF for 5 day. F4 and F7, transgenic lines overexpressing $\mathrm{BjFeCh}$ (BjFeCh-OE). Scale bars: 3.2 and $2.5 \mathrm{~cm}$ for the control and norflurazon, respectively.

and F7, respectively (Table 1 ).

To examine the effect of NF-induced photobleaching on the function of the photosynthetic apparatus, we measured the $F_{\mathrm{v}} / F_{\mathrm{m}}$ ratio, which indicates the maximum quantum efficiency of PSII photochemistry. ${ }^{20)}$ In response to NF, $F_{\mathrm{v}} / F_{\mathrm{m}}$ in WT plants decreased by 23\%, whereas transgenic lines F4 and F7 did not show a significant decline in $F_{\mathrm{v}} / F_{\mathrm{m}}$ (Fig. 3A). The $\phi_{\text {PSII }}$, the quantum yield of electron transport through PSII, decreased significantly upon NF treatment in WT and transgenic plants, with a greater decrease in WT (Fig. 3B). During NF-induced photobleaching, Stern-Volmer quenching increased 3.1- and twofold, respectively, in WT and transgenic plants, which is indicative of NPQ (Fig. 3C). To evaluate impairment of PSI and PSII, we performed quantitative analysis of photosynthetic protein complexes in the thylakoid membranes at the protein and gene expression levels by immunoblot analysis and qRT-PCR. The inhibition of carotenoid biosynthesis in NF-treated WT plants led to the disappearance of Lhca2 and Lhca3 proteins in PSI and Lhcb1 and Lhcb2 proteins in PSII (Fig. 4A). Although Lhca and Lhcb proteins decreased in transgenic plants in response to NF, the remaining Lhca and Lhcb levels were still observed during photobleaching, indicating partial maintenance of the structural integrity of the photosynthetic apparatus. In response to NF, ex- 


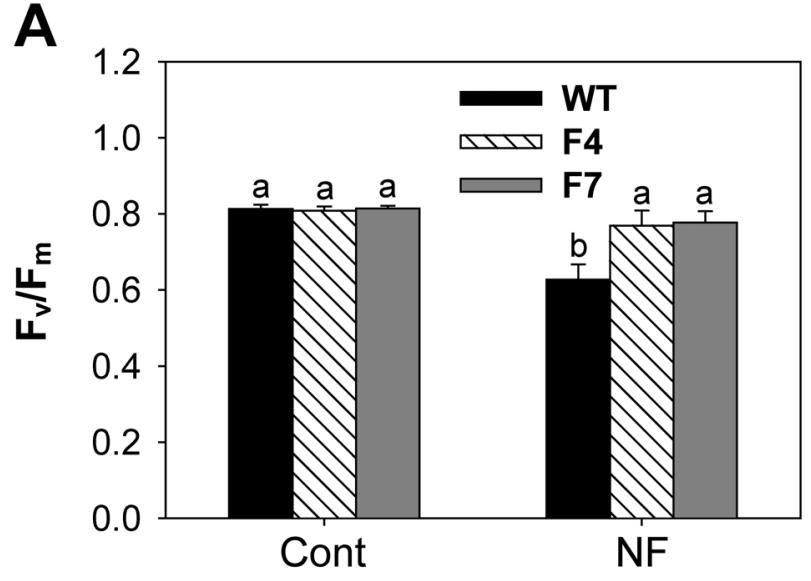

B
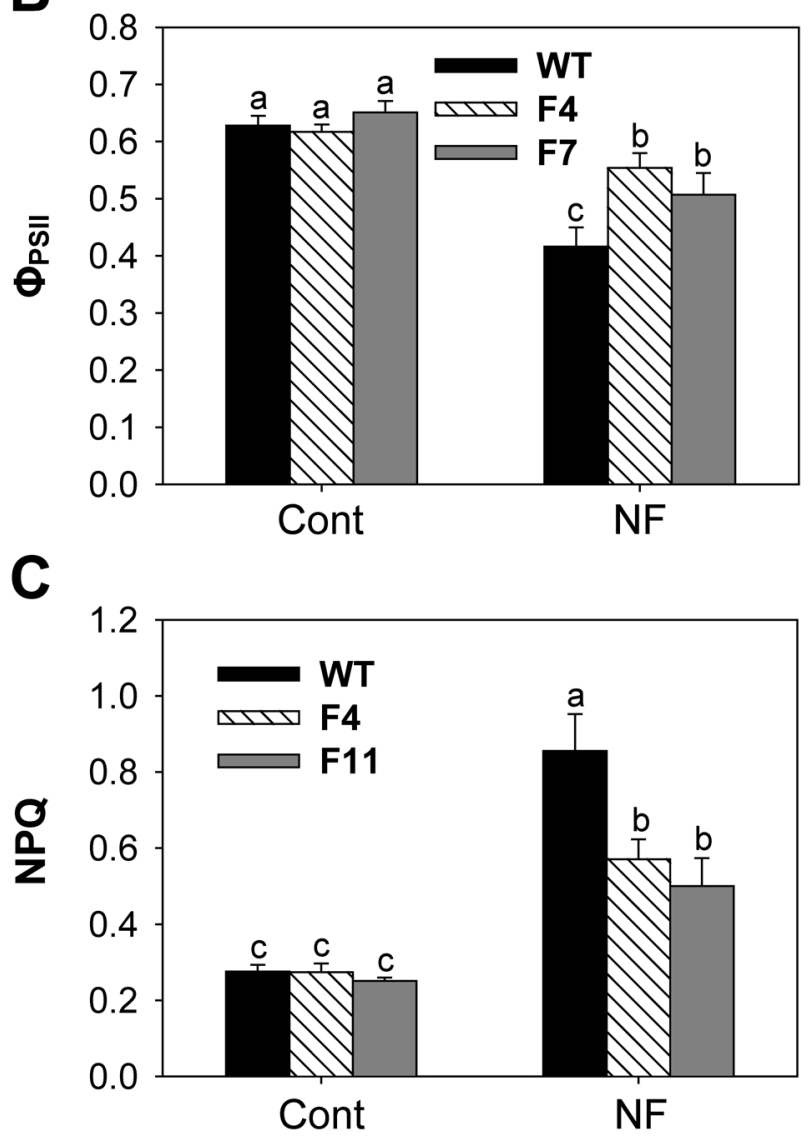

Fig. 3. Function of photosystems in WT and transgenic rice treated with norflurazon. $F_{\mathrm{v}} / F_{\mathrm{m}}$, efficiency of excitation capture by open PSII reaction centers (A), $\phi_{\text {PSII }}$; the quantum yield of electron transport through PSII (B), and NPQ (C). Plants were subjected to the same treatments as in Fig. 2. Cont, control; NF, norflurazon treatment; WT, non-transgenic wild type; F4 and F7, transgenic lines expressing BjFeCh. Values are the means \pm S.E. from three independent experiments. Means with the same letter are not significantly different at $p<0.05$ by LSD test.

pression levels of $L h c b 1$ and $L h c b 2$, the genes for Lhcb proteins of PSII, greatly decreased in WT and transgenic plants, with a greater decrease in WT plants (Fig. 4B), which correlated with the result of Lhcb proteins.
2. Effect of BjFeCh expression on porphyrin intermediates during NF-induced photobleaching

We compared metabolic changes in the porphyrin biosynthetic pathway of WT and transgenic plants during NF-induced photobleaching. The level of heme, which is a product of FeCh, was higher in controls of transgenic lines F4 and F7 than in WT plants (Fig. 5A). The heme content in both WT and transgenic plants decreased in response to NF; however, the levels of heme in F4 and F7 plants were 36\% and 47\% higher, respectively, than those in WT plants (Fig. 5A).

The end products of the chlorophyll branch, chlorophylls $(a+b)$, markedly decreased in NF-treated WT and transgenic plants, with a greater decrease in WT (Fig. 5B). In untreated control plants, the level of Proto IX, the branch-point precursor for both heme and chlorophyll, was similar in WT and transgenic plants (Fig. 6A). Levels of Mg-porphyrins, including MgProto IX, Mg-Proto IX ME, and Pchlide, were also similar in both plants (Fig. 6B-D). However, in response to NF, levels of Proto IX and $\mathrm{Mg}$-porphyrin intermediates decreased more in WT plants than in transgenic plants.

\section{Regulation of porphyrin biosynthesis genes during NF-induced photobleaching}

To assess alterations in the expression level of the transgene $\mathrm{BjFeCh}$ during NF-induced photobleaching, we examined the transcript level of $\mathrm{BjFeCh}$ by qRT-PCR. In transgenic lines F4 and F7, the transcript level of $\mathrm{BjFeCh}$ with NF treatment was not significantly different from that of untreated controls, whereas BjFeCh was not expressed in WT plants (Fig. 7A). Although the mRNA level did not change in response to NF, an increase in $\mathrm{BjFeCh}$ protein was observed in NF-treated transgenic plants (Fig. 7B).

To characterize the effects of $\mathrm{BjFeCh}$ expression on the porphyrin pathway, we analyzed the expression profiles of genes in porphyrin biosynthesis during NF-induced photobleaching. In untreated controls, the expression levels of genes involved in 5-aminolevulinate (ALA) synthesis were similar in WT and transgenic plants (Fig. 8A, B). OsHEMA1 encoding glutamyltRNA reductase was downregulated in NF-treated WT and transgenic plants, with greater downregulation in WT, whereas glutamate 1-semialdehyde aminotransferase (GSA) was maintained following NF treatment in transgenic plants. By contrast, the transcript levels of protoporphyrinogen oxidase 1 (OsPPO1), encoding the enzyme that produces Proto IX, increased significantly in WT and transgenic plants during NF-induced photobleaching (Fig. 8C).

In the heme branch, the transcript levels of endogenous $\mathrm{OsFeCh} 2$ in control plants did not differ significantly between WT and transgenic plants (Fig. 8D). In response to NF, WT and transgenic plants downregulated expressions of endogenous

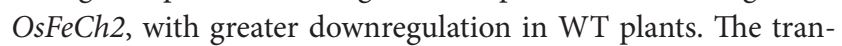
script levels of heme oxygenase (OsHO1) and OsHO2 in untreated controls were higher in transgenic plants than in WT plants (Fig. 8E, F). NF-induced photobleaching downregulated $\mathrm{OsHO1}$ 
A

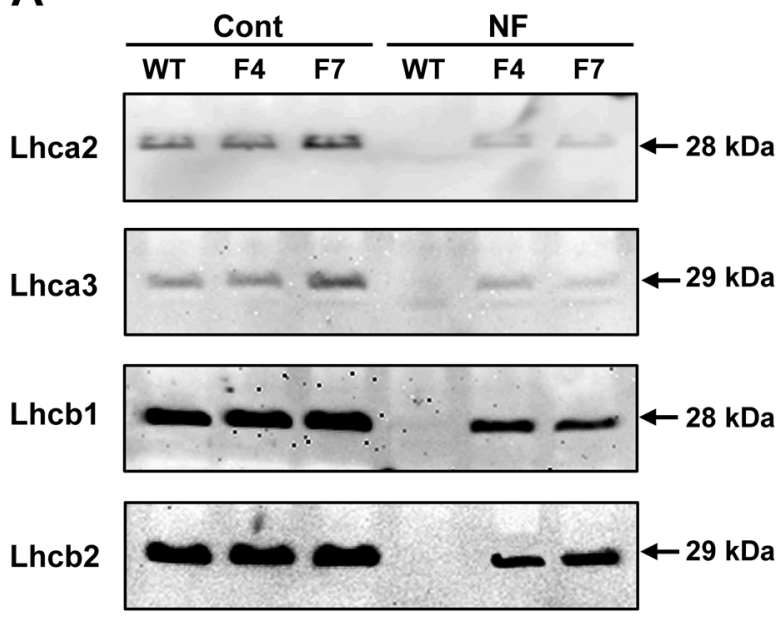

B

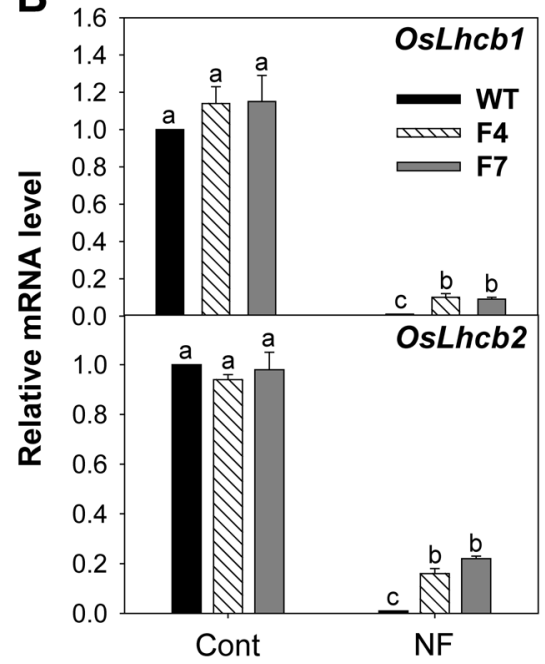

Fig. 4. Structure of photosystems by immunoblot analysis of the light-harvesting chlorophyll-binding proteins of PSI (Lhca2 and Lhca3) and PSII (Lhcb1 and Lhcb2) (A) and expression analysis of Lhcb genes by qRT-PCR (B) in WT and transgenic rice treated with norflurazon. The WT control was used for normalization, with the expression level of the sample set to 1 . The plants were subjected to the same treatments as in Fig. 2. Treatment notations are the same as in Fig. 3. Values are the means \pm S.E. from three independent experiments. Means with the same letter are not significantly different at $p<0.05$ by LSD test.

and OsHO2 in WT and transgenic plants, with greater downregulation in WT.

In untreated controls, the transcript levels of genes in the chlorophyll branch were similar in WT and transgenic plants (Fig. 8G-I). In response to NF, the transcript levels of $\mathrm{OsCHLH}$ and $\mathrm{OsCHLI}$ encoding the Mg-chelatase subunits decreased less in transgenic plants than in WT plants (Fig. 8G, H). The transcript levels of protochlorophyllide oxidoreductase B (OsPORB), encoding the enzyme that generates chlorophyllide from Pchlide, were downregulated in WT and transgenic plants, with greater downregulation in WT plants (Fig. 8I).

\section{Discussion}

The upregulation of $\mathrm{FeCh}$ was induced by NF treatment ${ }^{17,18)}$ and other stress conditions, such as paraquat, ozone, wounding, and drought. ${ }^{7,18,25,26)}$ In response to NF treatment, photobleaching

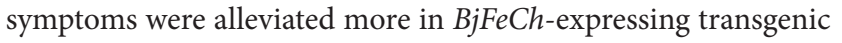
lines F4 and F7 than in WT plants (Fig. 2), which was correlated to the lesser reduction of carotenoid content (Table 1). In response to NF, the $F_{\mathrm{v}} / F_{\mathrm{m}}$ did not show a significant decline in transgenic lines F4 and F7, whereas $\phi_{\text {PSII }}$ decreased significantly in WT and transgenic plants, with a greater decline in WT plants (Fig. 3). The decline in $F_{\mathrm{v}} / F_{\mathrm{m}}$ by various abiotic stresses, including high light and herbicides, indicates the sensitivity of PSII activity.,27)

The NF-induced increase in NPQ was greater in WT plants than in transgenic plants (Fig. 3C), which is consistent with the increased photoinactivation of PSII, as indicated by a greater reduction in $F_{\mathrm{v}} / F_{\mathrm{m}}$. NPQ is proportional to the effective rate constant for energy dissipation in the antennae as well as the concentration of the quencher state. ${ }^{9,16)}$ The NF-induced disappearance of Lhca2 and Lhca3 proteins in PSI, as well as Lhcb1
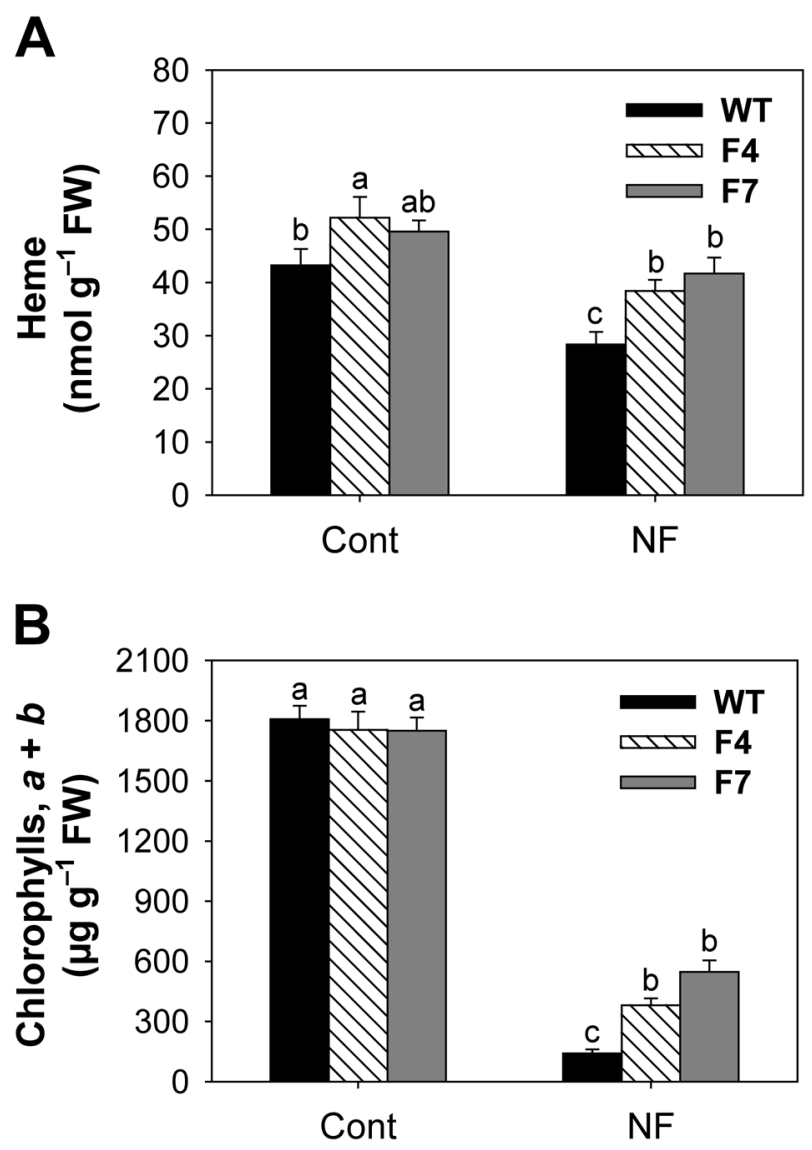

Fig. 5. Effect of norflurazon on heme (A) and chlorophylls $(a+b)(\mathrm{B})$ in WT and transgenic plants. The plants were subjected to the same treatments as in Fig. 2. Treatment notations are the same as in Fig. 3. Values are the means \pm S.E. from three independent experiments. Means with the same letter are not significantly different at $p<0.05$ by LSD test. 
A

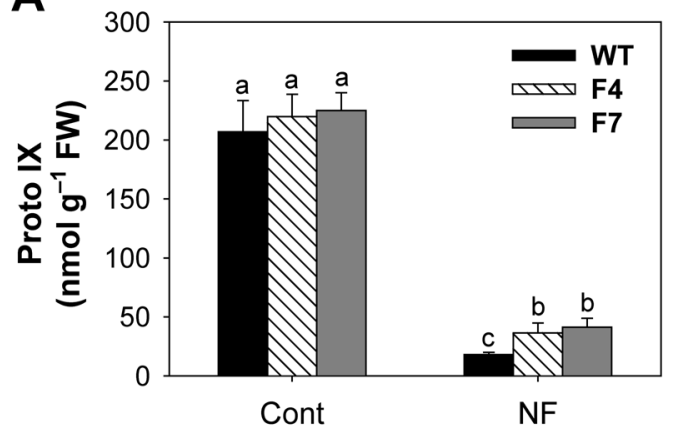

C

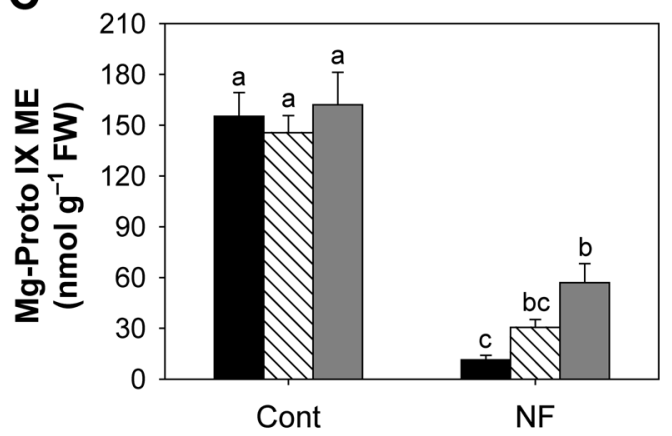

B

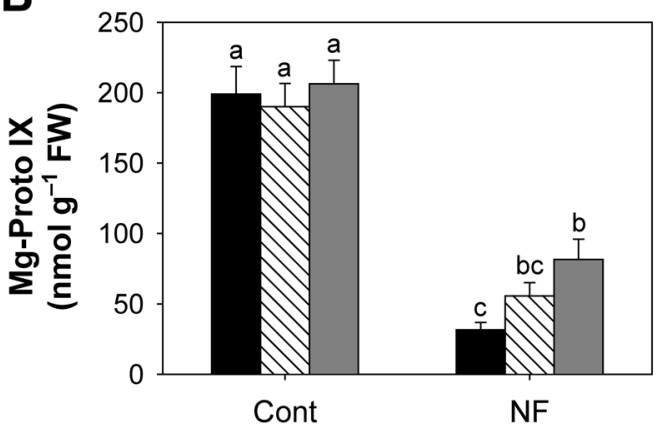

D

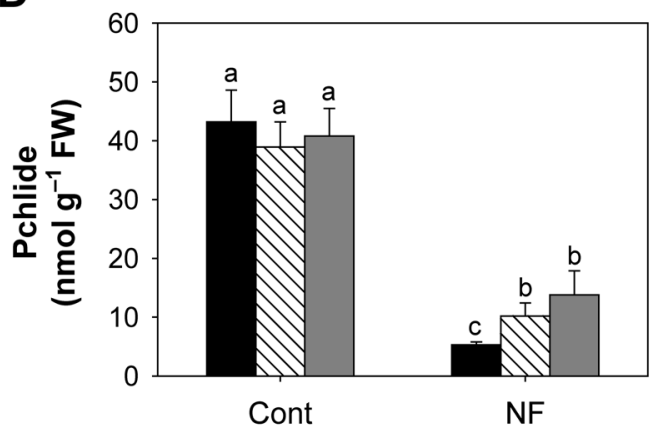

Fig. 6. Distribution of porphyrin metabolites in WT and transgenic plants treated with norflurazon. Proto IX (A), Mg-Proto IX (B), Mg-Proto IX ME (C), and Pchlide (D). Plants were subjected to the same treatments as in Fig. 2. Treatment notations are the same as in Fig. 3. Values are the means \pm S.E. from three independent experiments. Means with the same letter are not significantly different at $p<0.05$ by LSD test.

and Lhcb2 proteins in PSII, indicates severe impairment of PSI and PSII in WT plants (Fig. 4). When NF-treated plants are short of carotenoids, chlorophyll-binding proteins cannot be stabilized. ${ }^{28)}$ The remaining Lhc levels in NF-treated transgenic plants showed partial maintenance of the structural integrity of the photosynthetic apparatus, which may be related to sustained levels of carotenoids (Table 1). In response to NF, the smaller decline in the expression levels of Lhcb1 and Lhcb2 in transgenic plants than in WT plants (Fig. 4B) was correlated with the result of Lhcb proteins, indicating that Lhcb expression is regulated at the transcription level. Our results demonstrate that transgenic rice expressing $\mathrm{BjFeCh}$ alleviates NF-induced photobleaching by sustaining higher levels of carotenoid, $F_{\mathrm{v}} / F_{\mathrm{m}}, \phi_{\mathrm{PSII}}$, and Lhc pro- teins, as compared to WT plants. The expression of BjFeCh also mitigated necrotic symptoms caused by the peroxidizing herbicide acifluorfen in transgenic rice. ${ }^{19)}$ These results suggest a possible role of $\mathrm{FeCh}$ in protection against various types of stresses, including photobleaching and necrosis.

The expression of $\mathrm{BjFeCh}$ in transgenic plants led to increased levels of heme in untreated controls, as compared to that in WT plants (Fig. 5A). In response to NF, the heme content decreased in transgenic plants, but remained higher than in WT plants. During NF-induced photobleaching, the metabolic flow from Proto IX to heme appears to be higher in transgenic plants than in WT plants, thereby sustaining heme levels. The maintenance of heme biosynthesis has been suggested to be an important fac-
A

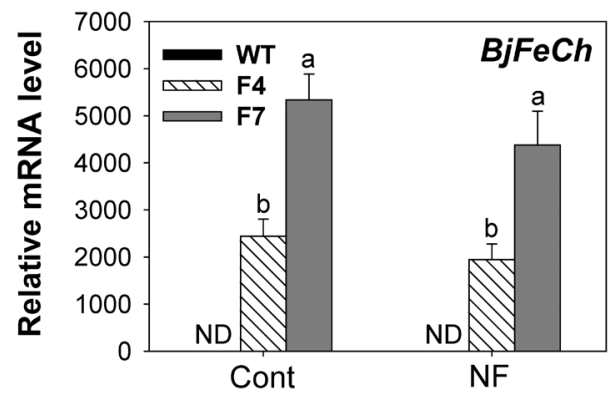

B

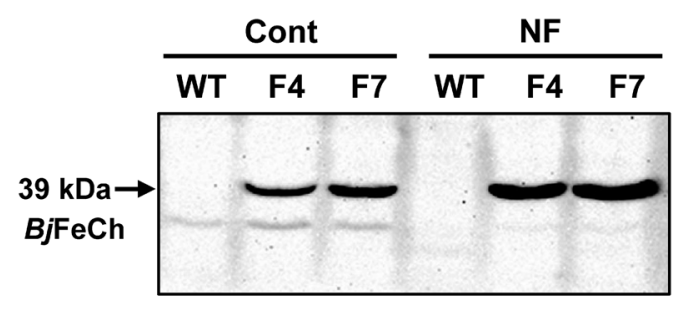

Fig. 7. Effect of norflurazon on the expression levels of BjFeCh mRNA (A) and BjFeCh protein (B). Plants were subjected to the same treatments as in Fig. 2. Treatment notations are the same as in Fig. 3. ND, not detected in WT. Values are the means \pm S.E. from three independent experiments. Means with the same letter are not significantly different at $p<0.05$ by LSD test. 


\section{Common pathway}
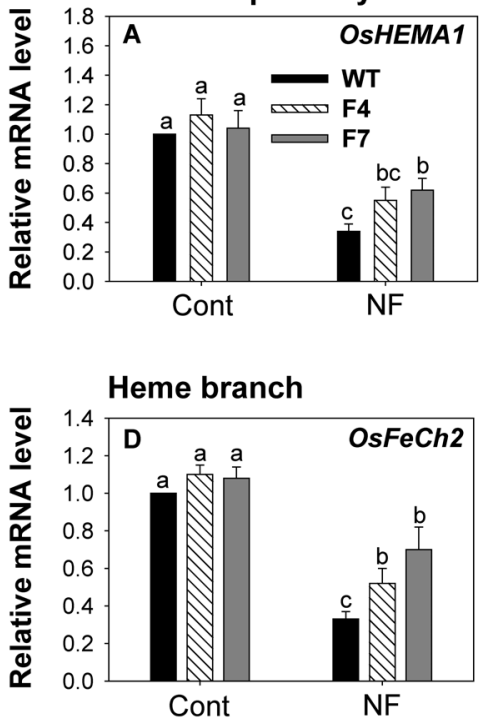

Chlorophyll branch

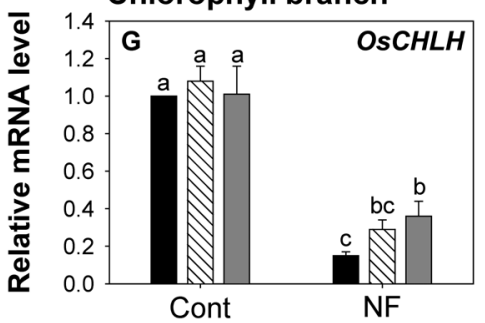

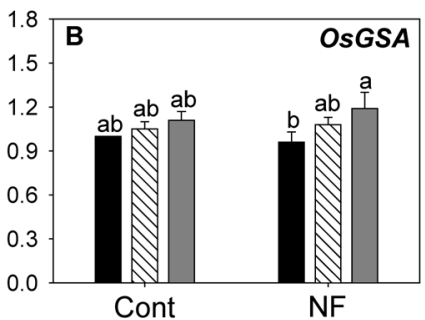
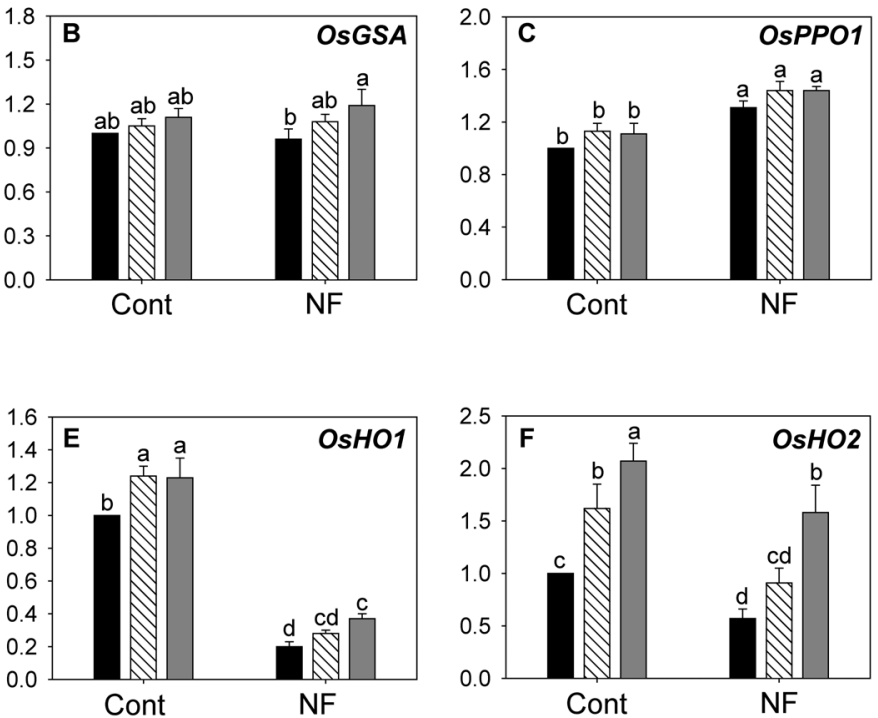

Cont

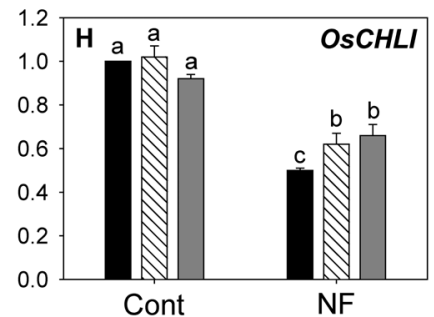

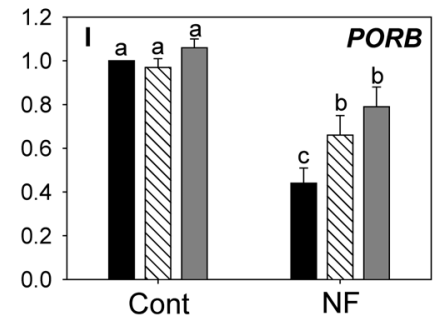

Fig. 8. Norflurazon-induced changes in the expression of genes encoding porphyrin biosynthesis enzymes in WT and transgenic plants. Common pathway $(A-C)$, heme branch $(D-F)$, and chlorophyll branch (G-I). Plants were subjected to the same treatments as in Fig. 2. Treatment notations are the same as in Fig. 3. The WT control was used for normalization, with the expression level of the sample set to 1. Values are the means \pm S.E. from three independent experiments. Means with the same letter are not significantly different at $p<0.05$ by LSD test.

tor for increasing resistance to drought stress and acifluorfen. ${ }^{7,19)}$ While the levels of heme remained quite high during photobleaching, chlorophylls $(a+b)$ markedly decreased in NF-treated WT and transgenic plants, with a greater decrease in WT plants (Fig. 5B). During NF-induced photobleaching, levels of Proto IX, Mg-Proto IX, Mg-Proto IX ME, and Pchlide showed greater decreases in WT plants than in transgenic plants (Fig. 6). Rapid decrease in porphyrin intermediates has also been observed under drought stress, which may result from porphyrin-degrad-

Table 1. Effects of norflurazon on total carotenoid content in wild-type (WT) and transgenic rice expressing $\mathrm{BjFeCh}$.

\begin{tabular}{ccc}
\hline \multirow{2}{*}{ Plant } & \multicolumn{2}{c}{ Carotenoid content $\left(\mu \mathrm{g} \mathrm{g}^{-1} \mathrm{FW}\right)$} \\
\cline { 2 - 3 } & Control & Norflurazon \\
\hline WT & $955 \pm 12 \mathrm{a}$ & $126 \pm 14 \mathrm{~d}$ \\
F4 & $944 \pm 31 \mathrm{a}$ & $280 \pm 16 \mathrm{c}$ \\
F7 & $931 \pm 30 \mathrm{a}$ & $363 \pm 34 \mathrm{~b}$ \\
\hline
\end{tabular}

The plants were subjected to the same treatments as in Fig. 2. Values are the means \pm S.E. from three independent experiments. Treatment notations are the same as in Fig. 2. Means with the same letter are not significantly different at $p<0.05$ by LSD test. ing processes. ${ }^{7)}$ During oxidative stress response, sensing of the porphyrin state of the cell induces the regulation of porphyrin homeostasis, diminishing the photosensitizing effects of porphyrin intermediates. ${ }^{2,7,13)}$

The early steps in porphyrin biosynthesis, starting from ALA, are required for the formation of heme and chlorophyll. ${ }^{1,4)}$ During NF-induced photobleaching, the downregulation of HEMA1 and GSA for ALA synthesis was greater in WT plants than in transgenic plants, whereas the transcript levels of PPO1 significantly increased in WT and transgenic plants (Fig. 8A-C). This indicates that chloroplastic PPO1 plays a key role in the stress response to NF-induced photobleaching. Similarly, the expression of PPO2, encoding the mitochondrial PPO2 isoform, was induced by NF treatment. ${ }^{17)}$ Unlike the NF-induced upregulation of $\mathrm{FeCh} 2$ in a previous study, ${ }^{17)} \mathrm{WT}$ and transgenic plants treated with NF downregulated endogenous OsFeCh2, with greater downregulation in WT plants (Fig. 8D). This discrepancy may be due to a 5 day prolonged exposure of NF-treated plants to light in our study, which causes severe photooxidative stress. After NF treatment, transgenic tobacco containing the $\mathrm{AtFeCh} 2$ reporter gene construct also downregulated AtFeCh2, whereas tobacco containing the $A t F e C h 1$ reporter gene up- 
regulated $\mathrm{AtFeCh} 1 .^{18)}$ In transgenic rice, the transcript level of $\mathrm{BjFeCh}$ during $\mathrm{NF}$ treatment was not significantly different from that of controls, whereas the expression levels of $B j \mathrm{FeCh}$ protein greatly increased in response to NF (Fig. 7), indicating that the NF-induced regulation of $B j \mathrm{FeCh}$ may occur at a translational step. These results imply that a sustained level of heme in NF-treated transgenic plants mainly derives from the markedly increased expression of $\mathrm{BjFeCh}$, not $\mathrm{OsFeCh}$. HO catalyzes the oxidation of heme and yields biliverdin IX $\alpha,{ }^{5)}$ which is known to be a potent antioxidant. ${ }^{29,30)}$ Higher expression levels of $\mathrm{HO1}$ and $\mathrm{HO} 2$ in controls of transgenic plants (Fig. 8E, F) may result from the upregulation of heme biosynthesis. Although NFinduced photobleaching downregulated $\mathrm{HO}$ and $\mathrm{HO} 2$ in WT and transgenic plants, higher expression levels of $H O$ in transgenic plants seem to play a role in mitigating photobleaching via biliverdin IX $\alpha$. In response to NF, transgenic plants showed lesser decreases in the transcript levels of CHLH, CHLI, and PORB as compared to those in WT plants (Fig. 8G-I). In NF-induced photobleaching, the reduction in porphyrin intermediates seems to be mainly due to inhibited plastid biogenesis as well as the necessity of downregulating the biosynthesis of phototoxic porphyrins. Overall, expression levels of genes in both chlorophyll and heme branches remained higher in transgenic plants than in WT plants during NF-induced photobleaching, which was consistent with higher levels of chlorophyll intermediates and heme.

Taken together, transgenic rice expressing $\mathrm{BjFeCh}$ increased resistance to NF-induced photobleaching, as indicated by sustained levels of carotenoid, chlorophyll intermediates, $F_{\mathrm{v}} / F_{\mathrm{m}}$, and Lhc proteins as compared to those in WT plants. This resistance is likely to be due to the NF-induced increase of the $B j \mathrm{FeCh}$ protein level in transgenic plants, which leads to higher levels of heme and $H O$ than in WT plants. As compared to WT plants, higher expression levels of chlorophyll biosynthetic genes in transgenic plants during photobleaching may also contribute to the maintenance of higher levels of chlorophylls and Lhc proteins, thereby mitigating the photodamage to photosynthetic complexes. This study provides not only insight into the strict regulation of the porphyrin biosynthetic pathway that occurs during NF-induced photobleaching, but also a useful transgenic tool to study the resistance mechanism of herbicide-resistant crops.

\section{Acknowledgements}

This research was supported by the Basic Science Research Program through the National Research Foundation of Korea (NRF) funded by the Ministry of Education (NRF-2018R1D1A1B07043814 and NRF2020R1I1A3073932). No conflicts of interest have been declared.

\section{References}

1) S. I. Beale and J. D. Weinstein: Tetrapyrrole metabolism in photosynthetic organisms. "Biosynthesis of Heme and Chlorophyll," ed. by H. A. Daily, McGraw-Hill, New York, pp. 287-391, 1990.

2) A. W. U. Busch and B. L. Montgomery: Interdependence of tetrapyrrole metabolism, the generation of oxidative stress and the mitigative oxidative stress response. Redox Biol. 4, 260-271 (2015).

3) R. M. Larkin: Tetrapyrrole signaling in plants. Front. Plant Sci 7, 1586 (2016).

4) R. Tanaka and A. Tanaka: Tetrapyrrole biosynthesis in higher plants. Annu. Rev. Plant Biol. 58, 321-346 (2007).

5) M. J. Terry, P. J. Linley and T. Kohchi: Making light of it: The role of plant heme oxygenases in phytochrome chromophore synthesis. Biochem. Soc. Trans. 30, 604-609 (2002).

6) F. Ricchelli: Photophysical properties of porphyins in biological membranes. J. Photochem. Photobiol. B 29, 109-118 (1995).

7) T.-H. Phung, H. I. Jung, J. H. Park, J. G. Kim, K. Back and S. Jung: Porphyrin biosynthesis control under water stress: Sustained porphyrin status correlates with drought tolerance in transgenic rice. Plant Physiol. 157, 1746-1764 (2011).

8) T.-H. Phung and S. Jung: Alterations in the porphyrin biosynthesis and antioxidant responses to chilling and heat stresses in Oryza sativa. Biol. Plant. 59, 341-349 (2015).

9) B. Demmig-Adams and W. W. Adams III: Photoprotection in an ecological context: the remarkable complexity of thermal energy dissipation. New Phytol. 172, 11-21 (2006).

10) E. H. Murchie and K. K. Niyogi: Manipulation of photoprotection to improve plant photosynthesis. Plant Physiol. 155, 86-92 (2011).

11) N. Nisar, L. Li, S. Lu, N. C. Khin and B. J. Pogson: Carotenoid metabolism in plants. Mol. Plant 8, 68-82 (2015).

12) P. Böger and G. Sandmann: Carotenoid biosynthesis inhibitor herbicides-mode of action and resistance mechanisms. Pestic. Outlook 9, 29-35 (1998).

13) J. H. Park, L. H. Tran and S. Jung: Perturbations in the photosynthetic pigment status result in photooxidation-induced crosstalk between carotenoid and porphyrin biosynthetic Pathways. Front. Plant Sci $\mathbf{8}$, 1992 (2017).

14) J.-H. Park and S. Jung: Perturbations of carotenoid and tetrapyrrole biosynthetic pathways result in differential alterations in chloroplast function and plastid signaling. Biochem. Biophys. Res. Commun. 482, 672-677 (2017).

15) A. M. Gilmore and H. Y. Yamamoto: Linear models relating xanthophylls and lumen acidity to non-photochemical fluorescence quenching. Evidence that antheraxanthin explains zeaxanthin-independent quenching. Photosynth. Res. 35, 67-78 (1993).

16) A. V. Ruban: Nonphotochemical chlorophyll fluorescence quenching: Mechanism and effectiveness in protecting plants from photodamage. Plant Physiol. 170, 1903-1916 (2016).

17) M. Moulin, A. C. McCormac, M. J. Terry and A. G. Smith: Tetrapyrrole profiling in Arabidopsis seedlings reveals that retrograde plastid nuclear signaling is not due to Mg-protoporphyrin IX accumulation. Proc. Natl. Acad. Sci. U.S.A. 105, 15178-15183 (2008).

18) D. P. Singh, J. E. Cornah, S. Hadingham and A. G. Smith: Expression analysis of the two ferrochelatase genes in Arabidopsis in different tissues and under stress conditions reveals their different roles in haem biosynthesis. Plant Mol. Biol. 50, 773-788 (2002).

19) J. G. Kim, K. Back, H. Y. Lee, H. J. Lee, T. H. Phung, B. Grimm and S. Jung: Increased expression of Fe-chelatase leads to increased metabolic flux into heme and confers protection against photodynamically induced oxidative stress. Plant Mol. Biol. 86, 271-287 (2014).

20) B. Genty, J. M. Briantais and N. R. Baker: The relationship between the quantum yield of photosynthetic electron-transport and quenching of chlorophyll fluorescence. Biochim. Biophys. Acta 990, 87-92 (1989).

21) W. Bilger and O. Björkman: Role of the xanthophyll cycle in photoprotection elucidated by measurements of light-induced absorbance 
changes, fluorescence and photosynthesis in leaves of Hedera canariensis. Photosynth. Res. 25, 173-185 (1990).

22) I. Lermontova and B. Grimm: Reduced activity of plastid protoporphyrinogen oxidase causes attenuated photodynamic damage during high-light compared to low-light exposure. Plant J. 48, 499-510 (2006).

23) H. K. Lichtenthaler: Chlorophylls and carotenoids: pigments of photosynthetic biomembranes. Methods Enzymol. 148, 350-382 (1987).

24) M. A. Schneegurt and S. I. Beale: Biosynthesis of protoheme and heme $a$ from glutamate in maize. Plant Physiol. 81, 965-971 (1986).

25) T. Avin-Wittenberg, V. Tzin, R. Angelovici, H. Less and G. Galili: Deciphering energy-associated gene networks operating in the response of Arabidopsis plants to stress and nutritional cues. Plant J. 70, 954-966 (2012).

26) S. Nagai, M. Koide, S. Takahashi, A. Kikuta, M. Aono, Y. SasakiSekimoto, H. Ohta, K. Takamiya and T. Masuda: Induction of iso- forms of tetrapyrrole biosynthetic enzymes, AtHEMA2 and AtFC1, under stress conditions and their physiological functions in Arabidopsis. Plant Physiol. 144, 1039-1051 (2007).

27) E. H. Murchie and T. Lawson: Chlorophyll fluorescence analysis: A guide to good practice and understanding some new applications. J. Exp. Bot. 64, 3983-3998 (2013).

28) O. Czarnecki, C. Gläßer, J. G. Chen, K. F. X. Mayer and B. Grimm: Evidence for a contribution of ALA synthesis to plastid-to-nucleus signaling. Front. Plant Sci 3, 236 (2012).

29) G. O. Noriega, K. B. Balestrasse, A. Batlle and L. Tomaro: Heme oxygenase exerts a protective role against oxidative stress in soybean leaves. Biochem. Biophys. Res. Commun. 323, 1003-1008 (2004).

30) G. G. Yannarelli, G. O. Noriega, A. Batlle and M. L. Tomaro: Heme oxygenase up-regulation in ultraviolet-B irradiated soybean plants involves reactive oxygen species. Planta 224, 1154-1162 (2006). 